\title{
A Longitudinal Study of Quality of Life and Psychological Functioning for Active, Fluctuating, and Inactive Disease Patterns in Inflammatory Bowel Disease
}

Lisa M. Lix ${ }^{1,5}$

Lesley A. Graff ${ }^{2,5}$

John R. Walker ${ }^{2,5}$

Ian Clara ${ }^{3,5}$

Patricia Rawsthorne $e^{4,5}$

Linda Rogala ${ }^{4,5}$

Norine Miller ${ }^{4,5}$

Jason Ediger ${ }^{4,5}$

Thea Pretorius ${ }^{6}$

Charles N. Bernstein ${ }^{4,5}$

${ }^{1}$ Department of Community Health Sciences, University of Manitoba, Winnipeg, CANADA

${ }^{2}$ Department of Clinical Health Psychology, University of Manitoba, Winnipeg, CANADA

${ }^{3}$ Department of Psychology, University of Manitoba, Winnipeg, CANADA

${ }^{4}$ Department of Internal Medicine, University of Manitoba, Winnipeg, CANADA

${ }^{5}$ University of Manitoba Inflammatory Bowel Disease Clinical and Research Centre, Winnipeg, CANADA

${ }^{6}$ Health, Leisure, and Human Performance Research Institute, University of Manitoba,

Winnipeg, CANADA

Address for Correspondence:

Lisa M. Lix, PhD PStat

Department of Community Health Sciences, University of Manitoba

408-727 McDermot Avenue

Winnipeg, MB R3E 3P5

CANADA

Phone: (204) 975-7799 Fax: (204) 789-3910

e-mail: lisa lix@cpe.umanitoba.ca

Short Title: Longitudinal Study of IBD Patterns

Abbreviations: AIC - Aikake Information Criterion; CD - Crohn's disease; IBD - inflammatory bowel disease; IBDQ - Inflammatory Bowel Disease Questionnaire; ICC - Intra-class correlation; QOL - Quality of life; SF-36 - Medical Outcomes Study 36-item Short Form Questionnaire; UC - ulcerative colitis 


\begin{abstract}
Objectives: To assess quality of life (QOL) and psychological functioning in inflammatory bowel disease (IBD) as related to patterns of disease activity over time. Methods: Study participants were 388 recently-diagnosed individuals from the population-based Manitoba IBD Cohort Study. They completed mail-out surveys at six-month intervals and clinical interviews annually. Based on their two-year pattern of self-reported disease activity, participants were assigned to one of three groups: consistently active, fluctuating, or consistently inactive disease. Disease type (Crohn's disease [CD] or ulcerative colitis [UC]) was confirmed through chart review. Change over time was modeled for measures of QOL and positive and negative psychological functioning using mixed-effects regression analyses. Results: Half of the participants had fluctuating disease activity, while almost one-third of participants reported consistently active disease. Participants with the fluctuating activity pattern showed significant improvement in disease-specific QOL compared to participants with consistent activity. Perceived stress, health anxiety, and pain anxiety decreased while pain catastrophizing and mastery increased over time, although the amount of change was not significantly different among disease activity patterns. However, when the data were averaged over time there were significant differences among disease activity patterns on most outcomes. Significant effects of CD versus UC were observed only for the pain measures. Conclusions: Change in IBD QOL is influenced by one's longitudinal profile of disease activity, but change in psychological functioning is not. Effects of disease activity on psychological functioning were modest, suggesting that disease has an impact even when patients are not experiencing active symptoms.
\end{abstract}

Abstract Word Count: 247 


\section{A Longitudinal Study of Quality of Life and Psychological Functioning for Active,}

\section{Fluctuating, and Inactive Disease Patterns in Inflammatory Bowel Disease}

\section{Introduction}

Inflammatory bowel disease (IBD) is a chronic illness characterized by periods of remission and disease exacerbation. Studies have shown that individuals with IBD have lower quality of life (QOL) than the general population, as well as diminished psychological functioning and well-being. ${ }^{1-4}$ Outcomes tend to be poorer during periods of active than of inactive disease. ${ }^{5-9}$ These observations have primarily been made in studies that focus on associations between disease activity and IBD outcomes at a single point in time.

Longitudinal studies can help to identify IBD outcomes that are sensitive to treatment interventions or changes in disease severity or activity. ${ }^{10-13}$ Research about the latter topic has been limited. Blondel-Kucharski et al. ${ }^{1}$ focused on changes in QOL for patients with Crohn's disease (CD) over a one-year period with assessments at three-month intervals. There was significant improvement in QOL over time which the authors attribute to lower rates of disease activity at study endpoint, although this association was not directly tested. Porcelli et al. ${ }^{14}$ compared patients with CD and ulcerative colitis (UC) at just two time points in a six-month period. Regardless of disease type, individuals for whom disease activity increased were more likely to report increased anxiety and depression while individuals for whom disease activity decreased over time reported reduced psychological distress.

Using data from the population-based Manitoba IBD Cohort Study, this study examines the trajectory of change for several measures of QOL and psychological functioning across multiple occasions in a two-year period. Based on previous research, we hypothesized that QOL and psychological functioning would be sensitive to disease activity over time, but that the 
trajectory of change would not differ for individuals with different types of disease (i.e., CD versus UC).

\section{Methods}

The Manitoba IBD Cohort Study is a prospective longitudinal study to identify the determinants of disease outcomes across multiple domains. The study was initiated in 2002, drawing on subjects from the University of Manitoba IBD Research Registry, a population-based registry established in 1995. A description of the methods used to establish the Registry has been

published previously, ${ }^{8}$ and is therefore not repeated here. The Cohort Study was approved by the University of Manitoba Health Research Ethics Board.

Individuals diagnosed within seven years (to capture relatively early disease) and who were 18 years of age or older $(N=606)$ were identified from the Registry and invited to join the study. A total of $418(69.0 \%)$ individuals agreed to participate. Following the initial contact, 19 individuals did not complete both the baseline survey and interview components, another four individuals withdrew, and seven individuals were found to be ineligible, resulting in a final sample of 388 participants for longitudinal follow-up. Data were collected using standardized self-report instruments distributed by mail at six-month intervals and interviews conducted by clinic staff at twelve-month intervals. This study focuses on data collected between the baseline wave (i.e., month 0) and the 24-month wave, giving a total of five measurement occasions. During this observation period, 34 subjects dropped out of the study, an attrition rate of $8.8 \%$.

Chart review by study staff was used to confirm patient report of disease type following the baseline wave of data collection. Self-report data on demographic characteristics (e.g., age, sex, ethnicity, marital status) were also collected at baseline. 
Disease activity was based on patient report of IBD symptom persistence for the previous six months using a six-point response format: "In the past six months my disease has been (a) constantly active, giving me symptoms every day (b) often active, giving me symptoms most days (c) sometimes active, giving me symptoms on some days (for instance 1-2 days/week) (d) occasionally active, giving me symptoms 1-2 days/month (e) rarely active, giving me symptoms on a few days in the past six months and (f) I was well in the past 6 months, what I consider a remission or absence of symptoms. Using a previously published $\operatorname{method}^{8}$, individuals were classified as having active or inactive disease at each measurement occasion. Active disease was defined as experiencing symptoms constantly to occasionally (i.e., response categories a through d), and inactive disease was defined as experiencing infrequent symptoms or feeling well (response categories e and f). Study participants were then assigned to one of three mutually exclusive groups based on their pattern of disease activity across the five measurement occasions: the consistently active disease group reported active disease at all measurement occasions, the consistently inactive disease group reported inactive disease at all measurement occasions, and the fluctuating disease activity group was composed of the remaining individuals. Standardized clinical indices of disease activity, the Harvey-Bradshaw for $\mathrm{CD}^{15}$ and PowellTuck for UC, ${ }^{16}$ were positively correlated with our six-point measure of disease activity (Spearman's $\rho=.40[p<.0001]$ for $\mathrm{CD} ; \rho=.50[p<.0001$ for UC at the baseline measurement occasion).

Study participants responded to a disease-specific QOL measure, the Inflammatory Bowel Disease Questionnaire $^{17}$ (IBDQ), as well as to a general QOL measure, the Medical Outcomes Study 36-item Short Form Questionnaire ${ }^{18}$ (SF-36). The IBDQ is the most commonly used and extensively validated QOL measure in IBD research. ${ }^{19}$ The 32 -item measure 
encompasses four domains: gastrointestinal symptoms, systemic problems, emotional dysfunction, and social difficulties. Total scores can range from 0 to 224, with higher scores indicating better QOL. The SF-36 assesses multiple dimensions of health. It provides separate physical and mental health component scores, each of which can range from 0 to 100 , with higher scores also indicating better QOL.

Five measures of negative psychological functioning were investigated: distress, perceived stress, health anxiety, pain anxiety, and pain catastrophizing. Higher scores indicate poorer functioning. Distress was assessed using the ten-item Kessler Psychological Distress Scale. ${ }^{20}$ This scale, which was specifically developed for use in epidemiologic surveys, provides a global measure of psychological distress based on questions about anxiety and depressive symptoms; scores range from 10 to 50, with scores less than 16 corresponding to reduced risk for an anxiety or depressive disorder. The Cohen Perceived Stress Scale is a 14-item questionnaire that has been validated as a tool to examine the role of stress in disease. ${ }^{21,22}$ Total scores range from 0 to 56. The Health Anxiety Questionnaire is a 21-item scale (total scores range from 0 to 63) measuring extent of health concerns and somatic focus. ${ }^{23}$ The 40 -item Pain Anxiety Symptom Scale assesses four dimensions of pain-related anxiety: cognitive, fear, escape/avoidance, and physiological; total scores range from 0 to $200{ }^{24}$ Finally, the Coping Strategies Questionnaire Catastrophizing subscale, on which scores can range from 0 to 28, was used to assess common maladaptive responses to pain. $^{25}$

Three measures of positive psychological functioning were also investigated: social support, well-being, and mastery. Higher scores indicate better functioning. The Multidimensional Scale of Perceived Social Support is a 12-item measure that assesses degree of support from friends and family. ${ }^{26}$ Total scores can range from 12 to 84 , and are usually re- 
scaled to range from 1 to 7 . Two measures of psychological resilience were adopted from a national health survey. The Psychological Well-being Manifestations Scale consists of 25 items that assess the individual's degree of a positive sense of themselves and their day-to-day functioning. ${ }^{27}$ Total scores range from 25 to 125 . Finally, the Mastery Scale assesses an individual's perceived control or efficacy; it has a possible range from 7 to $28 .^{28}$

In summary, longitudinal data were collected on 11 outcomes. However, not all measures were collected at each occasion because of the potential for participant fatigue due to the large number of response items. The three QOL measures, as well as perceived stress and social support were obtained at all five measurement occasions, while health anxiety, well-being, and mastery were collected at three occasions: 0,12 , and 24 months. The remaining measures were collected at two occasions: distress at 0 and 12 months, and pain anxiety and catastrophizing at 0 and 24 months.

Trend plots of the data were used to assess the assumption of a linear trajectory of change in the study outcomes and identify potential outliers/influential observations. Measures of skewness and kurtosis were computed to assess potential departures from a multivariate normal distribution. Correlations were computed to assess the pattern of association among the repeated measurements. Frequency distributions and logistic regression revealed that subject attrition was not associated with age group, disease type, or sex; we assume that the missing observations are missing at random, or ignorable. ${ }^{29}$

Linear mixed-effects regression analyses ${ }^{30-32}$ were used to test the trend in each of the outcomes over time (measured in months) and to model the data as a function of the fixed effects of pattern of disease activity and disease type, as well as random (i.e., subject-specific) effects. The confounding covariates of age and sex were included as fixed effects in all models. Mixed- 
effects models have been recommended for analyzing longitudinal QOL outcomes, in part because they can accommodate data that are unbalanced due to missing observations ${ }^{33}$ Regression parameters were estimated using maximum likelihood techniques.

The model initially fitted for each outcome included two-way interactions of time $\mathrm{x}$ disease type, time $\mathrm{x}$ disease activity pattern, and disease type $\mathrm{x}$ disease activity pattern. To ensure a parsimonious final model, interaction effects that were non-significant were excluded. A random intercept and a random slope for time were initially included in each model; the random slope for time was only retained in the final model when it resulted in a significant improvement in model fit and where model convergence was achieved. Model fit was assessed using the Aikake Information Criterion (AIC), a penalized log-likelihood measure. ${ }^{34}$ An independence covariance structure was fit to the model residuals because goodness of fit (as measured by the AIC) did not improve substantially for any of the outcomes when a model for correlated residuals (e.g., first-order autoregressive or compound symmetric structure) was adopted. All analyses were conducted using the MIXED procedure in SAS version 9.1. ${ }^{35}$

The output from the mixed-effect models includes $F$ tests of main and interaction effects as well as regression parameter estimates (i.e., $\hat{b}$ ) for the fixed effects, and their associated standard errors, $t$-statistics, and $p$-values. Tests of statistical significance were conducted at $\alpha=$ .05. The intra-class correlation (ICC), the proportion of variation in the outcome variable(s) explained by the random (i.e., subject-specific) effect(s), is also reported. ${ }^{31}$ The ICC ranges in value from 0 to 1 , with higher values indicating a greater proportion of total variation due to within-subject variation. 


\section{Results}

For the 388 study participants, the baseline chart review confirmed that $187(48.2 \%)$ had CD and 169 (43.6\%) had UC. Another $18(4.6 \%)$ were identified as having indeterminate colitis and $14(3.6 \%)$ did not have definite IBD. The latter two groups of individuals were excluded, leaving 356 participants for subsequent analyses. More than half of the study participants (59\%) were women. They were predominantly Caucasian (91\%), with small numbers having selfdescribed backgrounds such as East Indian, Hispanic, or Metis (of Aboriginal and European ancestry). The age range was from 18 to 83 years. For participants with $\mathrm{CD}$ the mean age was 38.5 years $(\mathrm{SD}=14.6)$ while for those with $\mathrm{UC}$ the mean age was 43.0 years $(\mathrm{SD}=14.7)$. Average duration of disease at baseline (in years) was $4.4(\mathrm{SD}=2.1)$ for participants with $\mathrm{CD}$ and $4.3(\mathrm{SD}=2.1)$ for those with $\mathrm{UC}$.

The number and percent of study participants with self-reported active and inactive disease at each measurement occasion is reported in Table 1 for CD and UC. Overall, 109 (30.6\%) participants were classified as having a consistently active disease pattern during the 24month observation period, 60 (16.9\%) were classified as having a consistently inactive disease pattern, and 174 (48.9\%) were classified as having a fluctuating disease activity pattern. The remaining $13(3.7 \%)$ could not be classified into one of these three groups because of missing data on disease activity, and were excluded from subsequent analyses. Descriptive statistics for the QOL and psychological functioning measures are reported in Table 2 for these three disease activity patterns at the baseline and 24-month measurement occasions; separate results are provided for CD and UC. 


\section{Assessments of $Q O L$}

Mixed-effects regression models for the QOL measures were conducted first. All models included a random intercept; retention of a random slope for time did not result in improved model fit as evaluated by the AIC.

For the IBDQ, there was a statistically significant disease activity pattern $\mathrm{x}$ time interaction $(F[2,1277]=6.65, p=.0013)$. No other interaction terms were statistically significant. Figure 1 illustrates the trajectory of change for the three patterns of disease activity; the regression coefficients for the interaction are reported in Table 3. Compared to study participants with consistently active disease, those with consistently inactive disease showed no significant change in IBDQ scores over time $(p=.1183)$, while those with fluctuating disease activity showed a small but significant improvement in IBDQ scores $(p=.0003)$. There was no significant main effect of disease type for the IBDQ $(F[1,1277]=0.60, p=.4391)$.

None of the interaction effects were statistically significant for the SF-36 mental health or physical health components. As well, the main effect of time was not significant for either component (mental health: $F[1,1197]=0.24, p=.6210$; physical health: $F[1,1197]=0.18, p=$ .6714). There was a significant main effect of disease activity pattern for the mental health component $(F[2,1197]=3.85, p=.0216)$, but not for the physical health component $(F[2,1197]$ $=1.01, p=.3661$ ). For the former (see Table 3 ), individuals with the pattern of consistently inactive disease had a significantly higher mean score than individuals with consistently active disease $(p=.0067)$. There was no significant main effect of disease type on either the mental health $(F[1,1197]=1.32, p=.8927)$ or physical health $(F[1,1197]=0.02, p=.8811)$ components. 
The ICC values for the QOL models are also reported in Table 3. For the IBDQ, slightly more than half of the total variation could be explained by the random intercept, which indicates that there was substantial subject-specific variation in the data. The ICC was much smaller for both SF-36 components.

Assessments of Negative Psychological Functioning

None of the two-way interactions was statistically significant in the models for the negative psychological functioning measures, therefore only main effects were retained. A random intercept was included in all models, but a random slope for time was not retained because it did not result in improved model fit.

A significant main effect of time was observed for perceived stress $(F[1,1260]=25.79, p$ $<.0001)$, health anxiety $(F[1,649]=93.63, p<.0001)$, pain anxiety $(F[1,656]=59.84, p<$ $.0001)$, and pain catastrophizing $(F[1,651]=23.52, p<.0001)$, but not for distress $(F[1,537]=$ $1.40, p=.2381)$. There was a significant decrease in perceived stress, health anxiety, and pain anxiety, but a significant increase in pain catastrophizing over time (Table 4).

A significant main effect of disease activity pattern was observed for distress $(F[2,537]=$ $10.77 ; p<.0001)$, perceived stress $(F[2,1260]=11.13, p<.0001)$, and health anxiety $(F[2,649]$ $=10.46, p<.0001)$, but not for pain anxiety $(F[2,656]=1.65, p=.1947)$ or catastrophizing $(F[2,651]=0.05, p=.9483)$. As the results in Table 4 reveal, study participants with the consistently inactive disease pattern had a significantly lower mean score than respondents with a consistently active disease pattern for distress, perceived stress, and health anxiety $(p<.0001$ for all measures). As well for distress, the fluctuating disease activity group had a significantly lower mean score than the consistently active group $(p=.0002)$. 
There was a significant main effect of disease type on pain anxiety $(F[1,656]=6.21, p=$ $.0129)$, and catastrophizing $(F[1,656]=4.53, p=.0337)$. Mean scores were significantly higher for CD than for UC participants on both measures (Table 4). ICC values were highest for distress and pain anxiety. However, the ICC exceeded 0.50 for all measures, indicating that more than half of the total variation in the data could be attributed to subject-specific variation.

\section{Assessments of Positive Psychological Functioning}

For the three measures of positive psychological functioning, none of the two-way interactions was statistically significant; therefore the final models retained only main effects. A random intercept was included in each model, but a random slope for time was not retained because it did not result in improved model fit.

There was a significant main effect of time for mastery $(F[1,652]=5.46, p=.0197)$, but not for social support $(F[1,1278]=0.08, p=.7837)$ or psychological well-being $(F[1,653]=$ $0.06, p=.8123$ ). An increasing trend over time was evident for mastery (Table 5), although the magnitude of the effect was small.

A significant main effect of disease activity pattern was observed for well-being $(F[1$, $653]=11.51, p<.0001)$ and mastery $(F[2,652]=7.27, p=.0008)$, but not for social support $(F[2,1278]=2.96, p=.0519)$. As Table 5 reveals, compared to the consistently active disease group, the consistently inactive disease group reported significantly higher mean scores for wellbeing $(p<.0001)$ and mastery $(p=.0002)$. As well, participants with a fluctuating pattern of disease activity had significantly higher mean scores for well-being compared to those with a consistently active disease pattern $(p=.0019)$.

There was no significant main effect of disease type on any of the measures of positive psychological functioning: social support $(F[1,1278]=2.79, p=.0950)$, well-being $(F[1,653]=$ 
$0.01, p=.9679)$, mastery $(F[1,652]=0.73, p=.3919)$. ICC values for the measures of positive psychological functioning ranged from 0.56 to 0.66 . These values indicate that for all measures, more than half of the total variation was due to the subject-specific effect.

\section{Discussion}

This longitudinal study evaluated multiple, important variables related to coping with IBD using survey and interview data collected over a two-year period. The findings of this longitudinal study are consistent with a growing number of studies that have identified disease activity as an important determinant of outcomes in persons with IBD, however our assessment at multiple occasions strengthens the validity of this association. ${ }^{5,7,36}$ An earlier paper by our group $^{8}$ that focused only on the baseline data from the Manitoba IBD Cohort Study demonstrated substantial differences in disease-specific QOL and positive and negative psychological functioning in groups with self-reported active and inactive disease. However, while that paper reported no effect of disease type, the current analyses revealed that for both pain anxiety and pain catastrophizing, there were differences between CD and UC participants even after controlling for differences in the pattern of disease activity. CD is likely associated with more pain (or less predictable pain) than UC. Patients with CD have higher health care utilization than patients with $\mathrm{UC},{ }^{37}$ one indicator of greater morbidity, possibly including pain. As individuals progress through the disease course, pain may become a more pronounced factor and maladaptive responses may be more likely to emerge. As well, pain may be more difficult to stabilize among individuals with CD than with UC. Physicians should be encouraged to manage pain early on and not assume that all pain will resolve easily with the prescribing of immunomodulatory therapy. If patients develop good coping and relief mechanisms early in the 
disease course, they may be less likely to experience anxiety and the cognitive reactivity that can further exacerbate pain.

As expected, the disease-specific measure of QOL was sensitive to different patterns of disease activity. The average score for study participants with consistently inactive disease was similar to that reported in this literature for individuals in remission. ${ }^{38}$ Study participants with fluctuating disease activity showed a significant improvement in disease-specific QOL, while those with active disease showed a relatively flat trajectory. However, there was no difference between the consistently active and consistently inactive groups in the magnitude of change over time. This may be partially attributable to the smaller sample size for the latter group, resulting in an increased standard error and reduced sensitivity to detect a difference between disease activity patterns.

Average scores for the SF-36 measures of physical and mental health were below normative values for the Canadian population. ${ }^{39}$ However, these measures remained largely unchanged over the two-year observation period, and could not always differentiate among individuals with different patterns of disease activity. While previous research has recommended the use of both disease-specific and general measures of QOL to characterize patients with IBD,${ }^{40}$ our study suggests that only the former are sufficiently sensitive to be recommended for use.

Only one measure of negative psychological functioning, distress, did not show a significant increase or decrease over time. However, data for this measure were only available for two of the five measurement occasions. There may not have been sufficient time to detect change in individual's perceptions of distress over the disease course. 
The strengths of this study include the sample selection technique, low rate of attrition, and method of analysis. The Manitoba IBD Cohort Study draws participants from a large geographic area and with a diverse demographic profile. The population-based design helps to ensure the sample is representative of a broad range of individuals with IBD. Study attrition was less than $10 \%$ over the two-year observation period and drop-out was not concentrated in any particular cohort sub-group. These findings help to support the conclusion that the observed trajectories of change are representative of those expected in the IBD population. Unlike conventional longitudinal analysis techniques, mixed-effects models account for the inherent variability both among and within individuals. ${ }^{41}$ Our analyses revealed that there was substantial subject-specific variation on all of the measures, particularly the negative psychological functioning measures.

One potential limitation of this study is that it relies on a self-report measure to assign respondents to disease activity patterns. However, previous research has also used self-reports of disease symptoms and periods of remission to characterize disease activity in IBD populations. ${ }^{3}$ The feasibility of obtaining clinical measures of inflammation at six-month intervals in such a large sample of patients is low. Another limitation of the study is that respondents were assigned to one of only three groups based on their disease activity pattern; for example, in the fluctuating disease activity group there was no distinction between individuals who reported disease activity at all but one measurement occasion and those who reported disease inactivity at all but one measurement occasion. Subsequent investigations could explore these sub-groups, to investigate their trajectories of change in IBD outcomes. ${ }^{42}$

While this study described and tested the longitudinal change in measures of QOL and psychological functioning and their association with disease activity pattern and disease type, it 
does not test the causal mechanism of these relationships. Increasingly, the multivariate analysis technique of structural equation modeling is being applied in cross-sectional and longitudinal observational studies to test causal theories about health outcomes. ${ }^{43}$

This study only tested the change in total scores for each outcome measure. Previous cross-sectional studies have examined respondent differences on the components of the IBDQ and SF-36. ${ }^{5,40,44}$ This approach could be extended to longitudinal data, to investigate changes in different aspects of quality of life and their association with disease activity patterns and disease type. Studies of longitudinal change in other outcomes, such as health care use and factors associated with disease flares, could also be pursued.

In summary, having IBD impacts on patients' QOL, perceived stress, health anxiety, pain anxiety, pain catastrophizing, and mastery over time. Many of the measures used in this study could be adopted in clinical trials of IBD treatment efficacy given their responsiveness to disease activity and change over time. Nevertheless, the findings do raise some question about using the SF-36 in such studies because of its low discriminative performance. Patients with persistently active disease may have a greater need for psychological care in dealing with their emotional response to illness. Improvements in positive and negative psychological functioning over time were modest suggesting that disease is having an impact on people's lives even when they are not experiencing active symptoms. Fortunately, those with an active pattern did not show deterioration in quality of life and psychological functioning in spite of ongoing symptoms; rather, they tended to experience poorer functioning that persisted over time. 
Longitudinal Study of IBD Patterns

\section{References}

1. Blondel-Kucharski F, Chircop C, Marquis P, et al. Health-related quality of life in Crohn's disease: A prospective longitudinal study in 231 patients. Am J Gastroenterol 2001;96:29152920.

2. Guthrie E, Jackson J, Shaffer J, et al. Psychological disorder and severity of inflammatory bowel disease predict health-related quality of life in ulcerative colitis and Crohn's disease. Am J Gastroenterol 2002;97:1994-1999.

3. Petrak F, Hardt J, Clement T, et al. Impaired health-related quality of life in inflammatory bowel diseases: Psychosocial impact and coping styles in a national German sample. Scand J Gastroenterol 2001;36:375-382.

4. Porcelli P, Zaka S, Centonze S, et al. Psychological distress and levels of disease activity in inflammatory bowel disease. Ital J Gastroenterol 1994;26:111-115.

5. Casellas F, Arenas JI, Baudet JS, et al. Impairment of health-related quality of life in patients with inflammatory bowel disease: A Spanish multicenter study. Inflamm Bowel Dis 2005;11:488-496.

6. Casellas F, Lopez-Vivancos J, Casado A, et al. Factors affecting health related quality of life of patients with inflammatory bowel disease. Qual Life Res 2002;11:775-781.

7. Gibson PR, Weston AR, Shann A, et al. Relationship between disease severity, quality of life and health-care resources in a cross-section of Australian patients with Crohn's disease. J Gastroenterol Hepatol 2007; Epub ahead of print.

8. Graff LA, Walker JR, Lix LM, et al. The relationship of inflammatory bowel disease type and activity to psychological functioning and quality of life. Clin Gastroenterol Hepatol 2006; 4:1491-1501. 
9. Han SW, McColl E, Barton JR, et al. Predictors of quality of life in ulcerative colitis: The importance of symptoms and illness representations. Inflamm Bowel Dis 2005;11:24-34.

10. Duffy, LC, Zielezny MA, Marshall JR, et al. Lag time between stress events and risk of recurrent episodes of inflammatory bowel disease. Epidemiology 1991;2:141-145.

11. Levenstein S, Prantera C, Varvo V, et al. Stress and exacerbation in ulcerative colitis: A prospective study of patients enrolled in remission. Am J Gastroenterol 2000;95:1213-1220.

12. Mardini HE, Kip KE, Wilson JW. Crohn's disease: A two-year prospective study of the association between psychological distress and disease activity. Dig Dis Sci 2004;49:492497.

13. North CS, Alpers DH, Helzer JE, et al. Do life events or depression exacerbate inflammatory bowel disease? A prospective study. Ann Intern Med 1991;114:381-386.

14. Porcelli P, Leoci C, Guerra V, et al. A longitudinal study of alexithumia and psychological distress in inflammatory bowel disease. J Psychos Res 1996;41:569-573.

15. Harvey RF, Bradshaw JM. A simple index of Crohn's disease activity. Lancet 1980;1:514.

16. Powell-Tuck J, Brown RL, Lennard-Jones JE. A comparison of oral prednisolone given as single or multiple daily doses for active proctocolitis. Scand J Gastroenterol 1978;13:833837.

17. Guyatt GH, Mitchell A, Irvine EJ, et al. A new measure of health status for clinical trials in inflammatory bowel disease. Gastroenterology 1989;96:804-810.

18. Ware JE, Sherbourne CD. The MOS 36-item short-form health survey (SF-36). I. Conceptual framework and item selection. Med Care 1992;30:473-483.

19. Sainsbury A, Heatley RV. Review article: Psychosocial factors in the quality of life of patients with inflammatory bowel disease. Aliment Pharmachol Ther 2005;21:499-508. 
20. Kessler RC, Andrews G, Colpe LJ, et al. Short screening scales to monitor population prevalences and trends in non-specific psychological distress. Psychol Med 2002;32:959976.

21. Cohen S, Kamarck, Mermelstein R. A global measure of perceived stress. J Health Soc Behav 1983;24:385-396.

22. Cohen S, Williamson GM. Perceived stress in a probability sample of the United States. In: Spacapan SS, Oskemp S, eds. The social psychology of health. Newbury Park, CA: Sage, 1988:31-67.

23. Luccock MP, Morley S. The health anxiety questionnaire. Br J Health Psychol 1996;1:137150.

24. McGracken LM, Zayfert C, Gross RT. The pain anxiety symptoms scale: Development and validation of a scale to measure the fear of pain. Pain 1992;50:67-73.

25. Rosenstiel AK, Keefee FJ. The use of coping strategies in chronic low back pain patients: Relationship to patient characteristics and current adjustment. Pain 1983;17:33-44.

26. Zimet GD, Dahlem NW, Zimet SG, et al. The multidimensional scale of perceived social support. J Pers Assess 1988;52:30-41.

27. Masse R, Poulin C, Dassa C, et al. Elaboration and validation of a tool to measure psychological well-being: WBMMS. Can J Public Health 1998;89:352-357.

28. Pearlin LI, Schooler C. The structure of coping. J Health Soc Behav 1978;19:2-21.

29. Little RJA, Rubin DB. Statistical analysis with missing data. New York: Wiley, 1987.

30. Cudek R. Mixed-effects models in the study of individual differences with repeated measures data. Multivariate Behav Res 1996;31:371-403.

31. Hedeker D, Gibbons RD. Longitudinal data analysis. Hoboken, NJ: Wiley, 2006. 
32. Sullivan S, Dukes KA, Losina E. Tutorial in biostatistics: An introduction to hierarchical linear modeling. Stat Med 1999;18:855-888.

33. Beacon HJ, Thompson SG. Multi-level models for repeated measurement data: Application to quality of life data in clinical trials. Stat Med 1996;15:2717-2732.

34. Akaike H. A new look at the statistical model identification. IEEE Transactions on Automatic Control 1974;AC-19:716 -723.

35. SAS Institute, Inc. SAS/Stat user's guide, version 9. Cary, NC: SAS Institute, Inc., 2004.

36. Sewitch MJ, Abrahamowicz M, Bitton A, et al. Psychological distress, social support, and disease activity in patients with inflammatory bowel disease. Am J Gastroenterol 2001;96:1470-1479.

37. Longobardi T, Bernstein CN. Health care resource utilization in inflammatory bowel disease. Clin Gastroenterol Hepatol 2006;4:731-43.

38. Irvine, EJ, Feagan B, Rochon J, et al. Quality of life: A valid and reliable measure of therapeutic efficacy in the treatment of inflammatory bowel disease. Gastroenterology 1994; 106:287-296.

39. Hopman WM, Towheed T, Anastassiades T, et al. Canadian normative data for the SF-36 health survey. CMAJ 2000;163: 265-271.

40. McColl E, Han SW, Barton JR, et al. A comparison of the discriminatory power of the Inflammatory Bowel Disease questionnaire and the SF-36 in people with ulcerative colitis. Qual Life Res 2004;13:805-811.

41. Fairclough DL, Gagnon D, Papadopoulos G. Planning analyses of quality-of-life studies: A case example with migraine prophylaxis. J Biopharm Stat 2004;14:31-51. 
42. Leffondre K, Abrahamowicz M, Regeasse A, et al. Statistical measures were proposed for identifying longitudinal patterns of change in quantitative health indicators. J Clin Epidem 2004;57:1049-1062.

43. Raudenbush SW. Comparing personal trajectories and drawing causal inferences from longitudinal data. Annu Rev Psychol 2001;52:501-525.

44. Bernklev T, Jahnsen J, Schulz T, et al. Course of disease, drug treatment and health-related quality of life in patients with inflammatory bowel disease 5 years after initial diagnosis. Eur J Gastroenterol Hepatol 2005;17:1037-1045. 


\section{Study Support}

This research was supported in part by an operating grant from the Manitoba Health Research

Council to the first author and an operating grant from the Canadian Institutes of Health

Research to the research team. LML is supported in part by a Canadian Institutes of Health

Research New Investigator Award and CNB is supported in part by a Crohn's and Colitis

Foundation of Canada Research Scientist Award. The conduct of this research and preparation of this manuscript was independent of these funding sources.

\section{Conflict of Interest}

There are no conflicts of interest.

\section{Guarantor}

The first author (LML) is guarantor of this submission. 
Table 1. Frequency (\%) of active and inactive disease at each measurement occasion

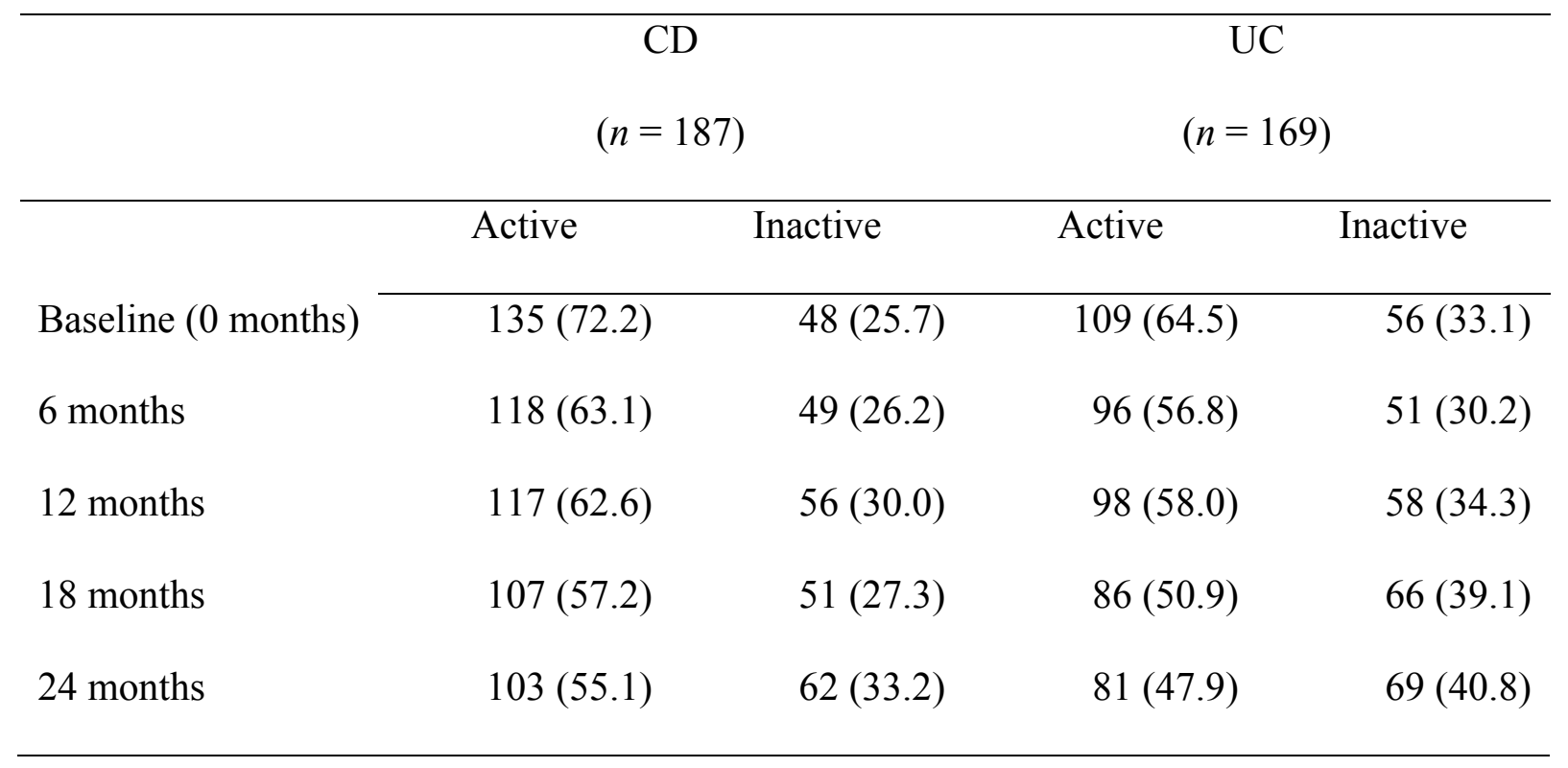

Note: row totals may not add to $N=356$ because of missing responses 
Table 2. Means (SDs) for quality of life and psychological functioning measures at baseline and 24 months for study participants classified by pattern of disease activity

\begin{tabular}{|c|c|c|c|c|c|c|}
\hline & & $\begin{array}{c}\mathrm{CD} \\
(n=187)\end{array}$ & & & $\begin{array}{c}\mathrm{UC} \\
(n=169)\end{array}$ & \\
\hline & Consistently & Fluctuating & Consistently & Consistently & Fluctuating & Consistently \\
\hline & Active Disease & Disease Activity & Inactive Disease & Active Disease & Disease Activity & Inactive Disease \\
\hline Quality of Li & & & & & & \\
\hline IBDQ & & & & & & \\
\hline 0 months & $152.9(26.7)$ & $165.4(31.5)$ & $184.2(23.6)$ & $160.0(29.4)$ & $168.5(31.1)$ & $189.4(20.8)$ \\
\hline 24 months & $155.0(31.5)$ & $180.2(23.4)$ & $193.3(20.4)$ & $163.0(27.5)$ & $180.6(23.2)$ & $198.7(16.9)$ \\
\hline SF-36 Menta & & & & & & \\
\hline $41.9(5.9)$ & $41.9(5.9)$ & $41.9(5.9)$ & $41.9(5.9)$ & $41.9(5.9)$ & $41.9(5.9)$ & $41.9(5.9)$ \\
\hline $41.1(5.9)$ & $41.1(5.9)$ & $41.1(5.9)$ & $41.1(5.9)$ & $41.1(5.9)$ & $41.1(5.9)$ & $41.1(5.9)$ \\
\hline SF-36 Physic & & & & & & \\
\hline 0 months & $41.9(5.5)$ & $43.4(4.2)$ & $42.7(6.3)$ & $42.4(5.3)$ & $42.3(4.7)$ & $44.0(3.6)$ \\
\hline 24 months & $43.0(5.4)$ & $44.3(3.7)$ & $41.8(3.3)$ & $41.7(4.4)$ & $42.6(4.5)$ & $43.5(2.4)$ \\
\hline
\end{tabular}




\begin{tabular}{|c|c|c|c|c|c|c|}
\hline & & $\begin{array}{c}\mathrm{CD} \\
(n=187)\end{array}$ & & & $\begin{array}{c}\mathrm{UC} \\
(n=169)\end{array}$ & \\
\hline \multicolumn{7}{|c|}{ Negative Psychological Functioning } \\
\hline \multicolumn{7}{|l|}{ Distress $^{\mathrm{a}}$} \\
\hline 0 months & $20.7(6.4)$ & $18.4(5.9)$ & $16.0(4.8)$ & $17.8(6.0)$ & $17.4(5.5)$ & $15.4(4.0)$ \\
\hline 12 months & $21.2(6.9)$ & $17.9(6.4)$ & $16.4(4.6)$ & $17.4(5.1)$ & $17.1(5.3)$ & $15.4(4.4)$ \\
\hline \multicolumn{7}{|c|}{ Perceived Stress } \\
\hline 0 months & $25.1(8.1)$ & $22.9(8.8)$ & $20.1(7.0)$ & $23.2(7.5)$ & $22.3(7.6)$ & $17.6(6.9)$ \\
\hline 24 months & $23.8(9.3)$ & $21.2(7.7)$ & $17.0(8.2)$ & $20.3(7.7)$ & $20.6(8.4)$ & $15.2(7.7)$ \\
\hline \multicolumn{7}{|c|}{ Health Anxiety } \\
\hline 0 months & $17.7(9.5)$ & $16.7(10.9)$ & $12.2(9.1)$ & $14.1(7.7)$ & $14.1(9.4)$ & $9.0(5.4)$ \\
\hline 24 months & $14.6(9.6)$ & $10.5(7.3)$ & $8.1(8.5)$ & $12.9(8.7)$ & $10.6(8.2)$ & $6.2(5.2)$ \\
\hline \multicolumn{7}{|c|}{ Pain Anxiety } \\
\hline 0 months & $79.8(27.0)$ & $74.4(30.6)$ & $72.0(31.1)$ & $67.6(30.7)$ & $67.8(27.8)$ & $61.2(26.1)$ \\
\hline 24 months & $74.3(30.8)$ & $64.9(27.9)$ & $59.7(29.2)$ & $56.8(27.8)$ & $63.7(30.3)$ & $41.5(22.2)$ \\
\hline
\end{tabular}




\begin{tabular}{|c|c|c|c|c|c|c|}
\hline & & $\begin{array}{c}\mathrm{CD} \\
(n=187)\end{array}$ & & & $\begin{array}{c}\mathrm{UC} \\
(n=169)\end{array}$ & \\
\hline \multicolumn{7}{|c|}{ Catastrophizing } \\
\hline 0 month & $13.2(4.6)$ & $12.7(4.0)$ & $12.2(5.0)$ & $11.1(4.6)$ & $12.4(3.8)$ & $12.0(5.8)$ \\
\hline 24 month & $14.7(5.3)$ & $14.1(5.1)$ & $14.0(5.4)$ & $12.5(5.8)$ & $13.1(4.9)$ & $12.8(4.4)$ \\
\hline \multicolumn{7}{|c|}{ Positive Psychological Functioning } \\
\hline \multicolumn{7}{|c|}{ Social Support } \\
\hline 0 months & $5.4(1.2)$ & $5.6(1.3)$ & $5.7(1.1)$ & $5.4(1.1)$ & $5.4(1.2)$ & $5.8(0.9)$ \\
\hline 24 months & $5.4(1.5)$ & $5.6(1.3)$ & $5.9(1.0)$ & $5.3(1.5)$ & $5.5(1.3)$ & $5.8(1.3)$ \\
\hline \multicolumn{7}{|l|}{ Well-Being } \\
\hline 0 months & $73.2(17.2)$ & $76.9(15.4)$ & $82.1(14.1)$ & $74.6(18.0)$ & $78.7(16.6)$ & $85.8(13.2)$ \\
\hline 24 months & $71.4(18.9)$ & $78.5(14.8)$ & $84.1(11.6)$ & $75.5(15.5)$ & $77.6(16.6)$ & $85.0(15.8)$ \\
\hline \multicolumn{7}{|l|}{ Mastery } \\
\hline 0 months & $18.0(5.0)$ & $19.2(4.4)$ & $19.3(4.2)$ & $19.2(3.4)$ & $19.9(4.9)$ & $21.9(3.7)$ \\
\hline 24 months & $18.6(5.0)$ & $20.1(4.0)$ & $19.3(4.4)$ & $19.8(4.3)$ & $19.9(5.6)$ & $22.3(4.2)$ \\
\hline
\end{tabular}


${ }^{a}$ Distress was not collected at the 24-month wave. The possible range of scores for each measure are as follows: IBDQ: 0 to 224; SF36 Physical Health: 0 to 100; SF-36 Mental Health: 0 to 100; Distress: 10 to 50; Perceived Stress: 0 to 56; Health Anxiety: 0 to 63; Pain Anxiety: 0 to 200; Catastrophizing: 0 to 28; Social Support: 1 to 7; Well-Being: 25 to 125; Mastery: 7 to 28. 
Table 3. Regression parameter estimates from mixed-effects regression models for quality of life measures

\begin{tabular}{|c|c|c|c|c|c|c|}
\hline \multirow{2}{*}{ Model Effect } & \multicolumn{2}{|c|}{ IBDQ } & \multicolumn{2}{|c|}{ SF-36 Mental Health } & \multicolumn{2}{|c|}{ SF-36 Physical Health } \\
\hline & $\hat{b}$ & se & $\hat{b}$ & se & $\hat{b}$ & se \\
\hline Time & 0.08 & 0.09 & 0.01 & 0.01 & 0.004 & 0.01 \\
\hline \multicolumn{7}{|l|}{ Disease Activity Pattern } \\
\hline Consistently Inactive & $31.89^{*}$ & 4.04 & $1.62 *$ & 0.60 & 0.48 & 0.50 \\
\hline Fluctuating & $10.49 *$ & 3.05 & 0.34 & 0.45 & 0.52 & 0.38 \\
\hline Consistently Active & Ref & -- & Ref & -- & Ref & -- \\
\hline \multicolumn{7}{|l|}{ Disease Type } \\
\hline Crohn's Disease & -1.94 & 2.51 & -0.47 & 0.41 & -0.05 & 0.35 \\
\hline Ulcerative Colitis & Ref & -- & Ref & -- & Ref & -- \\
\hline \multicolumn{7}{|l|}{ Time x Disease Activity Pattern } \\
\hline Time $\mathrm{x}$ Consistently Inactive & 0.24 & 0.15 & \multicolumn{2}{|c|}{$\mathrm{n} / \mathrm{a}$} & \multicolumn{2}{|c|}{$\mathrm{n} / \mathrm{a}$} \\
\hline Time $\mathrm{x}$ Fluctuating & $0.42 *$ & 0.11 & \multicolumn{2}{|c|}{$\mathrm{n} / \mathrm{a}$} & \multicolumn{2}{|c|}{$\mathrm{n} / \mathrm{a}$} \\
\hline Time x Consistently Active & Ref & -- & \multicolumn{2}{|c|}{$\mathrm{n} / \mathrm{a}$} & \multicolumn{2}{|c|}{$\mathrm{n} / \mathrm{a}$} \\
\hline ICC & \multicolumn{2}{|c|}{0.58} & \multicolumn{2}{|c|}{0.34} & \multicolumn{2}{|c|}{0.34} \\
\hline
\end{tabular}


Note: all parameter estimates are adjusted for age and sex; se $=$ standard error; $\mathrm{ICC}=$ intraclass correlation; $\mathrm{n} / \mathrm{a}$ indicates time $\mathrm{x}$ disease activity interaction was not included in the SF-36 mental and physical health models; * indicates a parameter estimate that is significant at $\alpha=.05$; a positive value of $\hat{b}$ for the time effect indicates that the average score increased over time while a negative value indicates that the average score decreased over time; a positive value of $\hat{b}$ for disease activity pattern or disease type indicates that the average score was higher than the reference group average score, while a negative value indicates that the average score was lower than the reference group average score. 
Table 4. Regression parameter estimates from mixed-effects regression models for negative psychological functioning measures

\begin{tabular}{|c|c|c|c|c|c|c|c|c|c|c|}
\hline \multirow{2}{*}{ Model Effect } & \multicolumn{2}{|c|}{ Distress } & \multicolumn{2}{|c|}{ Perceived Stress } & \multicolumn{2}{|c|}{ Health Anxiety } & \multicolumn{2}{|c|}{ Pain Anxiety } & \multicolumn{2}{|c|}{ Catastrophizing } \\
\hline & $\hat{b}$ & $\mathrm{Se}$ & $\hat{b}$ & se & $\hat{b}$ & se & $\hat{b}$ & se & $\hat{b}$ & se \\
\hline Time & -0.01 & 0.01 & $-0.08 *$ & 0.02 & $-0.15^{*}$ & 0.02 & $-0.34 *$ & 0.04 & $0.05 *$ & 0.01 \\
\hline \multicolumn{11}{|l|}{ Disease Activity Pattern } \\
\hline Consistently Inactive & $-3.80 *$ & 0.84 & $-5.08 *$ & 1.08 & $-5.78^{*}$ & 1.27 & -7.62 & 4.21 & -0.17 & 0.64 \\
\hline Fluctuating & $-1.93 *$ & 0.63 & -1.51 & 0.81 & -1.55 & 0.96 & -2.56 & 3.18 & 0.02 & 0.49 \\
\hline Consistently Active & Ref & -- & Ref & -- & Ref & -- & Ref & -- & Ref & -- \\
\hline \multicolumn{11}{|l|}{ Disease Type } \\
\hline Crohn's Disease & 0.76 & 0.58 & 1.19 & 0.75 & 1.13 & 0.88 & $7.27 *$ & 2.92 & $0.95 *$ & 0.45 \\
\hline Ulcerative Colitis & Ref & -- & Ref & -- & Ref & -- & Ref & -- & Ref & -- \\
\hline ICC & \multicolumn{2}{|c|}{0.74} & \multicolumn{2}{|c|}{0.58} & \multicolumn{2}{|c|}{0.69} & \multicolumn{2}{|c|}{0.76} & \multicolumn{2}{|c|}{0.57} \\
\hline
\end{tabular}

Note: all parameter estimates are adjusted for age and sex; se $=$ standard error; ICC $=$ intraclass correlation; * indicates a parameter estimate that is significant at $\alpha=.05$; a positive value of $\hat{b}$ for the time effect indicates that the average score increased over time while a negative value indicates that the average score decreased over time; a positive value of $\hat{b}$ for disease activity pattern or disease type indicates that the average score was higher than the reference group average score, while a negative value indicates that the average score was lower than the reference group average score. 
Table 5. Regression parameter estimates from mixed-effects models for positive psychological functioning measures

\begin{tabular}{|c|c|c|c|c|c|c|}
\hline \multirow{2}{*}{ Model Effect } & \multicolumn{2}{|c|}{ Social Support } & \multicolumn{2}{|c|}{ Well-Being } & \multicolumn{2}{|c|}{ Mastery } \\
\hline & $\hat{b}$ & se & $\hat{b}$ & se & $\hat{b}$ & $\mathrm{se}$ \\
\hline Time & 0.001 & 0.002 & -0.0 & 0.03 & $0.02 *$ & 0.01 \\
\hline \multicolumn{7}{|l|}{ Disease Activity Pattern } \\
\hline Consistently Inactive & 0.41 & 0.18 & $10.37 *$ & 2.22 & $2.41 *$ & 0.63 \\
\hline Fluctuating & 0.23 & 0.13 & $5.21 *$ & 1.67 & 0.93 & 0.48 \\
\hline Consistently Active & Ref & -- & Ref & -- & Ref & -- \\
\hline \multicolumn{7}{|l|}{ Disease Type } \\
\hline Crohn's Disease & 0.08 & 0.12 & 0.06 & 1.53 & -0.38 & 0.44 \\
\hline Ulcerative Colitis & Ref & -- & Ref & -- & Ref & -- \\
\hline ICC & \multicolumn{2}{|c|}{0.63} & \multicolumn{2}{|c|}{0.56} & \multicolumn{2}{|c|}{0.66} \\
\hline
\end{tabular}

Note: all parameter estimates are adjusted for age and sex; ICC = intraclass correlation; a positive value of $\hat{b}$ for the time effect indicates that the average score increased over time while a negative value indicates that the average score decreased over time; a positive value of $\hat{b}$ for disease activity pattern or disease type indicates that the average score was higher than the reference group average score, while a negative value indicates that the average score was lower than the reference group average score. 
Figure 1. Profile plot of means and $95 \%$ confidence intervals for the IBD quality of life measure

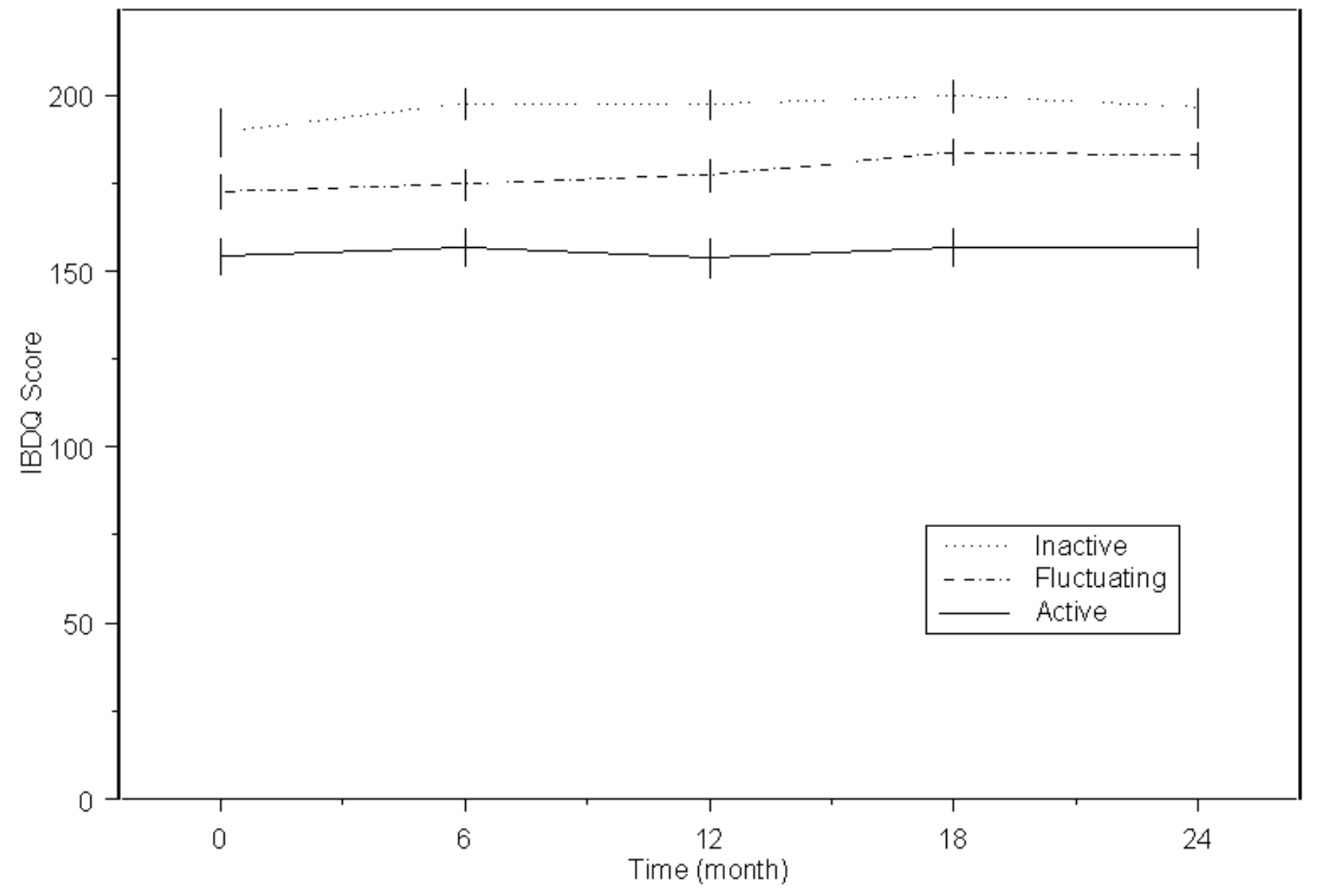

